
Not for reproduction, distribution or commercial use.

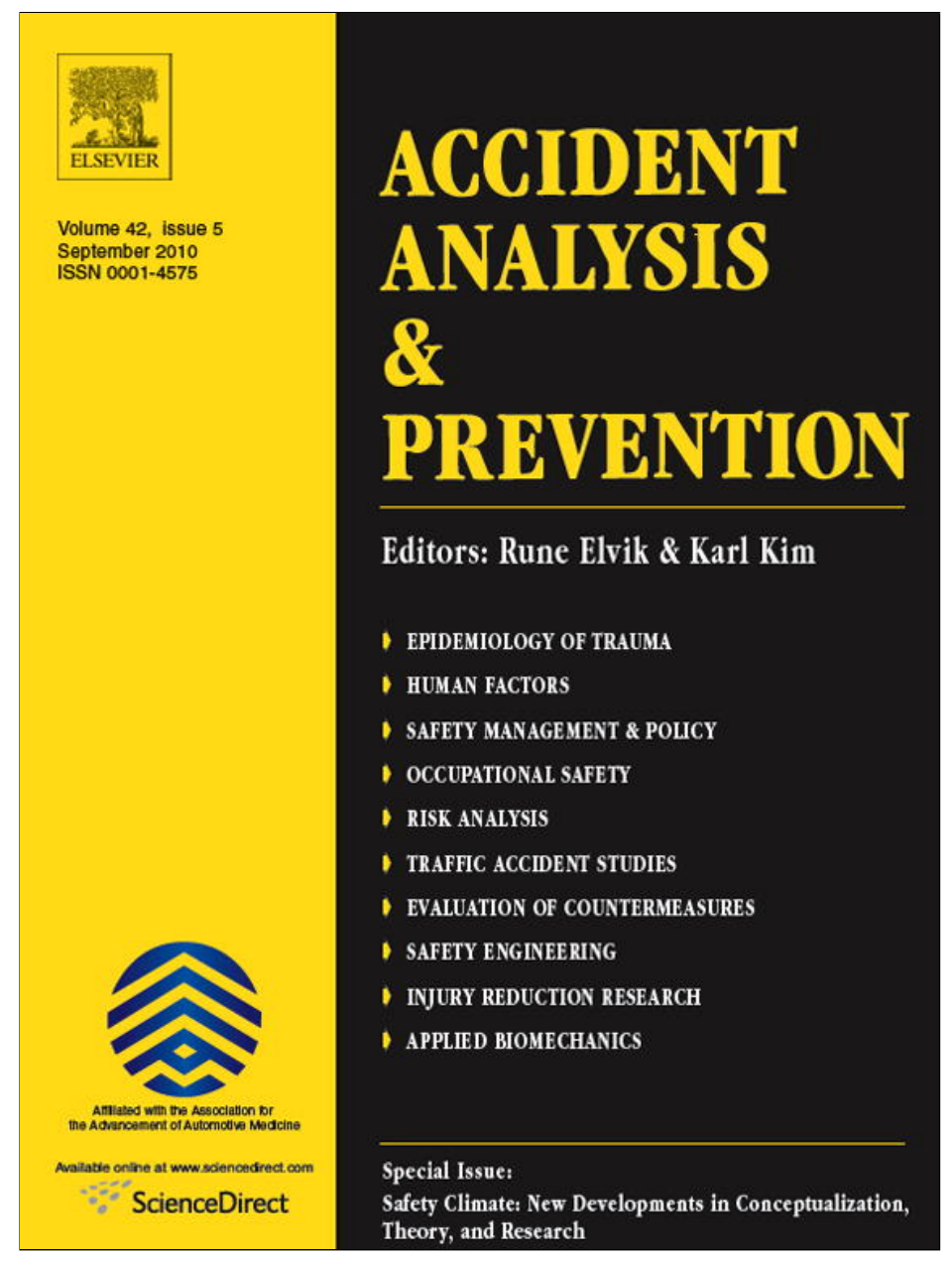

This article appeared in a journal published by Elsevier. The attached copy is furnished to the author for internal non-commercial research and education use, including for instruction at the authors institution and sharing with colleagues.

Other uses, including reproduction and distribution, or selling or licensing copies, or posting to personal, institutional or third party websites are prohibited.

In most cases authors are permitted to post their version of the article (e.g. in Word or Tex form) to their personal website or institutional repository. Authors requiring further information regarding Elsevier's archiving and manuscript policies are encouraged to visit:

http://www.elsevier.com/copyright 


\title{
Perceived colleagues' safety knowledge/behavior and safety performance: Safety climate as a moderator in a multilevel study
}

\author{
Li Jiang ${ }^{\mathrm{a}, \mathrm{b}}$, Guangtao $\mathrm{Yu}^{\mathrm{c}}$, Yongjuan $\mathrm{Li}^{\mathrm{a}}{ }^{\mathrm{*}}$, Feng $\mathrm{Li}^{\mathrm{a}}$ \\ a Institute of Psychology, Chinese Academy of Sciences, 10A Datun Road, Beijing, PR China \\ ${ }^{\mathrm{b}}$ Graduate School of Chinese Academy of Sciences, Yuquan Road, Beijing, PR China \\ ${ }^{\mathrm{c}}$ Central University of Finance and Economics, 39 Xueyuan South Road, Beijing, PR China
}

\section{A R T I C L E I N F O}

\section{Article history:}

Received 30 September 2008

Received in revised form 1 August 2009

Accepted 20 August 2009

\section{Keywords:}

Safety climate

Safety performance

Multilevel

Descriptive norms

\begin{abstract}
A B S T R A C T
This study presented a model specifying the relationship of unit-level safety climate and perceived colleagues' safety knowledge/behavior (PCSK/B) to safety behavior (safety compliance and safety participation), as well as safety performance (injuries and near misses). PCSK/B, a measure of descriptive norms, was taken as a new individual-level predictor. Hierarchical linear modeling analyses indicated the significant cross-level interaction effects of unit-level safety climate and PCSK/B on safety behavior, i.e., the more positive the safety climate, the stronger effects PCSK/B has on safety behavior. The effect of PCSK/B on injuries was mediated by safety behavior. Implications for management and safety climate research were discussed.
\end{abstract}

(C) 2009 Elsevier Ltd. All rights reserved.

\section{Introduction}

Injuries and accidents in workplace have always been a major issue worldwide, including China. In 2006, for example, 112,822 workers lost their lives in 627,158 industrial accidents, which means 320 workers died each workday on average (State administration of work safety, 2007). In addition to the costs in human suffering and loss of lives, industrial accidents cost China's economy an estimated $2 \%$ GDP, about 250 billion RMB (State administration of work safety, 2005). The similar astonishing statistics data were also obtained in other countries (Zohar, 2000). However, despite the serious social and economic implications, organizational safety issues have remained outside the mainstream of management research (Fahlbruch and Wilpert, 1999; Hofmann et al., 1995; Shannon et al., 1997; Zohar, 2002), and the situation in China is even more serious.

Accident investigations have revealed that organizational and cultural factors, considered as new research interests after nuclear accident at Chernobyl in 1986 (Cox and Flin, 1998; Pidgeon, 1998), are underlying causal factors of accidents (Seo, 2005). Generally speaking, safety climate was used to explain the organizational factors (e.g., McDonald et al., 2000), or as part of the organizational factors (Hetherington et al., 2006), the manifestation and snap of safety culture (Mearns and Flin, 1999). Therefore, safety climate

\footnotetext{
* Corresponding author. Tel.: +86 1064837339 ; fax: +86 1064872070

E-mail addresses: jiangli@psych.ac.cn (L. Jiang), yugtsd@163.com (G. Yu), liyj@psych.ac.cn (Y. Li), lifeng@psych.ac.cn (F. Li).
}

was regarded as an important indicator of workplace safety performance (Clarke, 2006; Cooper and Phillips, 2004). It relates to shared perceptions with regard to safety policies, procedures and practices, of which formal policy is explicit, relating to overt statements and formal procedures, while enforced policy or enacted practices are tacit, derived from observing management patterns of action concerning key policy issues (managerial practices) (Flin et al., 2000; Griffin and Neal, 2000; Zohar, 1980, 2008).

Not only safety climate which resulting from employee perceptions regarding the actions of management (Hofmann and Stetzer, 1996), but also their peers' behavioral pattern concerning safety is likely to affect individuals' safety performance (Kozlowski and Klein, 2000); and the two influence processes are shown separately (Clarke and Ward, 2006). Individuals make real changes to the feelings and behaviors as a result of interaction with others who are perceived to be similar, desirable, expert or the majority, which is the process of social influence (Rashotte, 2007). The general colleagues around might become the important referent social group of the employees. Based on this, we proposed an individual variable orienting direct contextual influence, perceived colleagues' safety knowledge and behavior (PCSK/B), to measure the group social influence. Additionally, research on safety climate has rarely been conducted in non-Western contexts. Compared to Westerners, people in China, think holistically instead of being analytically (Nisbett et al., 2001), which is a typical character of eastern culture-concerning contextual factors rather than rules (Peng et al., 2005). They feel more obliged to worry about the harmony of the in-group rather than their personal interests (Markus and Kitayama, 1991; Oyserman et al., 2002). The different cultural 
values might make the local employees have different perceptions of the safety climate and PCSK/B. Thus, the objectives of the current study were to examine the cross-level (the individual PCSK/B and unit-level safety climate) effect on safety performance in Chinese context.

\subsection{Unit-level safety climate}

Safety climate can be conceptualized as a higher order or global factor involving perceptions of workplace safety-related attributes and the relative priority of safety with other competing goals (such as productivity and speed) (Griffin and Neal, 2000; Zohar and Luria, 2005). Researchers who examined the effect of multilevel constructs argue that unit-level safety climate exists in a single organization because of different group processes (Neal and Griffin, 2006).

Unit-level safety climate refers to the shared safety perceptions of the unit as a whole (Neal and Griffin, 2006) with shared unit properties in the nature of the structure. According to Kozlowski and Klein's (2000) typology, shared unit properties are hypothesized to originate in various individual psychological processes or behaviors, and to converge among unit employees.

Zohar (2000) and Zohar and Luria (2005) empirically proved that unit-level safety climate converged because of different leadership processes, as leaders of each unit were taking their effect by holding distinctive criteria with regard to priority of safety versus other competing goals. The direct supervisors' managerial practices shape employees' perceptions of safety management commitment of their unit, which was showed significantly associated with unsafe behaviors (including different types of errors and violations), actual accidents, as well as the emotional outcomes (such as satisfaction) (Hofmann and Mark, 2006; Hofmann and Stetzer, 1996; Wallace et al., 2006). Study on the safety-specific leadership showed transformational leadership can improve employees' perceived safety climate (Barling et al., 2002). So it is logical to argue that in the same organization, significant between-unit variance of safety perception can be resulted from the influence of different leadership processes, which make it necessary to theoretically explore the effect of unit-level safety climate on safety performance.

\subsection{Perceived colleagues' safety knowledge/behavior}

In the workplace, employees' perceptions might be influenced not only by managerial practices and formal policies and procedures, but also by colleagues in the same work-unit (Clarke and Ward, 2006; DeJoy et al., 2004). Hofmann and Stetzer (1996), for example, suggested that approaching intentions, which is the likelihood that a team member would approach another team member engaged in unsafe behavior, play a mediating role in the relationship between group process and unsafe behavior. Clarke and Ward (2006) indicated that a possible reason for the absence of safety climate mediation on the leader influence tactics (coalition)-safety participation relationship is that this influence on behavior depends upon pressure from other team members rather than from the leader.

The effect of supervisors and team members on individuals' safety-related perceptions is shown separately (Clarke and Ward, 2006; Zohar, 2008) as the former is from the hierarchical position (Clarke, 1999) and authority, while the latter is a social influence process (Lapinski and Rimal, 2005). The social influences affecting individual psychological processes were initially categorized into normative influence and informational influence by Deutsch and Gerard (1955). Based on that, Cialdini and Raymond (1990) distinguished descriptive norms from injunctive norms, which are important measures of social norms under social setting or group norms under organizational setting (e.g., Klein and Boster, 2006; Ehrhart and Naumann, 2004). Descriptive norms, referring to what is done, are beliefs and perception about what is actually done by most others in one's social group. And injunctive norms describing what ought to be done, are perceptions of what others approve or disapprove (Kallgren et al., 2000). Descriptive norms can affect people's behavior particularly when the norms are in the focus of attention (Cialdini and Raymond, 1990). In social setting, the effect of descriptive norms on individual behavior or intention has been proved in judgment (Deutsch and Gerard, 1955), shopping (Burnkrant and Cousineau, 1975), technology acceptance (Venkatesh and Davis, 2000), stereotype beliefs (Wittenbrink and Henly, 1996), etc. In workplace setting, similar effect has also been proved, for example, people would adopt an innovation after they learn from social others' (such as peers) successful experiences with the innovation (Rogers, 1986). One of the most pervasive determinants of an individual's behavior is the influence of those around him (Burnkrant and Cousineau, 1975). From the interactionism perspective, unit members with similar backgrounds are likely to be a credible referent group for individuals (Ashforth, 1985). Thus, colleagues' safety beliefs, habits and behavior as a measure of descriptive norms, are likely to play important role in workplace safety.

Furthermore, studies in other field have already demonstrated the effect of culture on the relationship between normative factors and behavioral intentions. Bagozzi et al. (2000), for instance, found that their Chinese participants exhibiting the strongest relationship between norms and behavioral intention in fast food consumption; Park and Levine (1999) showed that the normative factors were significantly associated with interdependent but not independent self-construal. So in Chinese organizational context, employees would pay relatively more attention to the unit members around them, and the perceptions of colleagues' safety habits and behavior acting as descriptive norms would influence their own safety behavior, which is why PCSK/B is used to measure the descriptive norms in current study.

\subsection{The relationship between unit-level safety climate, PCSK/B and safety performance}

Safety compliance and safety participations are two kinds of safety behaviors. Safety compliance refers to activities employees need to do in order to maintain workplace safety (Griffin and Neal, 2000). When employees do not obey the procedures and rules, their behaviors are labeled "unsafe activities" or "violations". Thus, unsafe activities and safety compliance behaviors are two ends of one dimension. Safety participation refers to voluntary safety behaviors (Griffin and Neal, 2000). Safety compliance would be part of work role, whereas safety participation includes behaviors beyond formal role. Like the concept of organizational citizenship behaviors (OCBs) (Smith et al., 1983), which refers to extra-role voluntary behaviors beneficial to the organization, safety participation is also called "safety citizenship behavior" (Hofmann et al., 2003). Regarding safety-related outcomes, injuries and near misses are often used by safety researchers. Near misses are defined as incidents that could have caused an injury but did not (Goldenhar et al., 2003). In the current study, safety behaviors and safety-related outcomes were both taken as safety performance indicators.

Recent researches supported the validity of unit-level safety climate in predicting unsafe behaviors and accidents (Hofmann and Stetzer, 1996; Wallace et al., 2006), safety motivation and then safety behavior (Neal and Griffin, 2006), as well as medication errors (Hofmann and Mark, 2006). From social exchange perspective, if priority of safety is valued by unit leaders and top managers (i.e., with positive safety climate), they would demonstrate their commitment toward safety and concern for employees. And then 
employees would behave in a safe manner for an implied obligation (Hofmann and Morgeson, 1999; Hofmann et al., 2003). Therefore, we generated the following hypothesis:

Hypothesis 1a. Unit-level safety climate is positively related to safety compliance and safety participation.

According to the focus theory of normative conduct, perceptions of what others do (descriptive norms) provide the most efficient and adaptive actions, especially in ambiguous situations (Lapinski and Rimal, 2005). In the work setting, colleagues' safety knowledge/behavior would provide a guide for employees. If employees believe their colleagues are working safely, they would do so in the same manner. Thus, the following hypothesis was developed:

Hypothesis 1b. PCSK/B is positively related to safety compliance and safety participation.

Unit-level safety climate reflects management commitment and leaders' value on priority of safety, providing a guide when facing competing goals, complex and ambiguous information in the workplace (Gonzalez-Roma et al., 2002; Zohar and Tenne-Gazit, 2008). Although safety climate reflecting leaders' messages and actions may reduce complexity and ambiguity, unit members "are still exposed to a variety of discrepant organizational information such as job performance versus promotion decisions" (Zohar and TenneGazit, 2008). Clarke and Ward (2006) found the effect of safety climate on safety participation was not significant and they suggested a possible reason is this effect on behavior depends on other team members. That is to say, PCSK/B might play an incremental role in providing another guide for safety behavior.

Within positive safety climate, safety behavior is valued, expected and rewarded in the unit, and if other members in the unit obey safety rules, and voluntarily participate in dealing safety issues, as a result, employees would be more likely to engage in such a behavior that is not only valued and beneficial, but also prevalent and adaptive (e.g., what others do) in the unit.

Therefore, within positive safety climate, employees would be more likely motivated by PCSK/B and displaying much more safety behavior, while in negative safety climate, safety performance was less emphasized; the effect of PCSK/B on employees' safety behavior would be weaker. The following hypothesis was made:

Hypothesis 2. Unit-level safety climate moderates the effect of PCSK/B on safety behavior; specifically, the more positive the safety climate, the stronger the effect of PCSK/B on safety behavior.

Finally, with improved safety compliance and participation, as defined, employees would comply to the procedures and rules and voluntarily participate safety meetings and give suggestions, so accident risk associated with unsafe practices and rule violations would be reduced, and potential risks might be resolved in advance. As Neal and Griffin (2006) found, improvements of safety behavior within groups were associated with a subsequent reduction in accidents and injuries. Therefore, we hypothesized below:

Hypothesis 3a. Employee's safety behavior (safety compliance and safety participation) is related to lower levels of workplace injuries and near misses.

Other researchers suggested that attitudes and perceptions can predict behavior (i.e., safety compliance and safety participation) (Probst and Brubaker, 2001). Accidents are outcomes of numerous factors, and the individual unsafe behavior is one of the most direct trigger factor (Reason, 1990). The empirical link between safety climate and micro-accidents at group level was also established (Zohar, 2000). It was hypothesized that:

Hypothesis 3b. Employees' safety behavior (safety compliance and safety participation) will mediate the relationship between PCSK/B and safety-related outcomes (injuries and near misses).

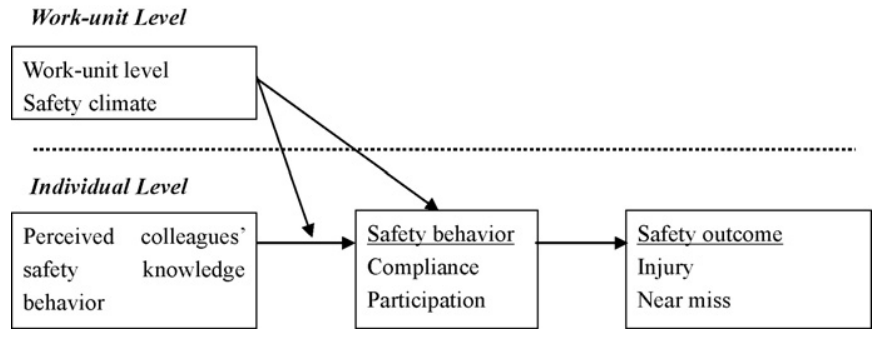

Fig. 1. A multilevel theoretical model of safety climate, safety performance and safety-related outcomes. The dash line separates work-unit level constructs and individual-level constructs.

The whole multilevel theoretical model illustrating the relationship among safety climate, safety performance and safety-related outcomes was depicted in Fig. 1.

\section{Methods}

\subsection{Participants}

Survey was administrated at 23 work-units (including polyester, decomposition, maintenance, etc.) of two petroleum and chemical corporations in China. Participants were all frontier employees. To control any differences between these two organizations, a dummy-coded variable was used in the analyses.

A total of 749 surveys were distributed with 631 valid responses returned, resulting a response rate of $84.2 \%$. A total of $61.9 \%$ participants were male. A majority of the participants $(75 \%)$ had a job tenure between 11 and 25 years. Gender was not significantly correlated with safety climate $(r=0.06), \mathrm{PCSK} / \mathrm{B}(r=0.05)$, safety compliance $(r=-0.03)$, safety participation $(r=-0.04)$. Tenure was not significantly correlated with safety climate $(r=-0.06)$, or PCSK/B $(r=0.00)$. There was low correlation between tenure and safety compliance $(r=0.10)$, safety participation $(r=0.20)$.

\subsection{Measures}

To ensure the equivalence of the measures in Chinese and English versions of the instrument, standard translation and backtranslation procedure (Brislin, 1980) were followed.

\subsubsection{Safety climate}

The items were mainly adapted from two safety climate scales: one was developed for railway industry (Glendon and Evans, 2007), and the other was validated in aviation (Evans et al., 2007). A total of 14 items were selected according to the characteristics of the energy industry. Employees responded on a 7-point scale to all items ranging from 1 (strongly disagree) to 7 (strongly agree). Safety training was evaluated by three items which were asked about the effectiveness of training for safety in the organization. An example of the items was "Training provides adequate skills and experience to carry out normal duties safely". Management commitment and communication for safety was evaluated by seven items that were asked about the degree to which managers were perceived to value priority of safety and the way in which safety issues were communicated. A sample item was "Management regard safety as an important part of operations." Safety equipment and maintenance was evaluated by four items that were asked about the way in which safety issues were maintained. A sample item was "Plant and equipment are maintained to a safe standard." To examine the structure of safety climate scale in our study, we randomly selected $50 \%$ of the data to conduct exploratory factor analysis (EFA), and the remaining data were submitted to confirmatory factor analysis (CFA). EFA results showed eigen values of one factor (accounting 
Table 1

Item loadings for exploratory factor analyses of safety climate and perceived colleagues' safety knowledge/behavior.

\begin{tabular}{|c|c|c|}
\hline Concept & Items & Factor \\
\hline \multirow{14}{*}{ Safety climate } & Management concerns staff's safety & 0.86 \\
\hline & Management were genuinely interested in safety issues & 0.83 \\
\hline & Management has a good understanding of operational issues that impact upon safety & 0.83 \\
\hline & Staff are able to openly discuss problems with supervisors or managers & 0.78 \\
\hline & Sufficient money are allocated for maintenance to be completed to an adequate standard & 0.77 \\
\hline & Staff who report issues are provided with timely feedback & 0.77 \\
\hline & Sufficient staff are allocated for maintenance to be completed to an adequate standard & 0.74 \\
\hline & Maintenance on reported faults is carried out in a timely manner & 0.72 \\
\hline & Management consult staff about safety issues & 0.70 \\
\hline & Training provides adequate skills and experience to carry out normal duties safely & 0.67 \\
\hline & Adequate training is received when new procedures or equipment are introduced & 0.63 \\
\hline & Management regard safety as an important part of operations & 0.61 \\
\hline & Regular training is provided for a range of emergency situations & 0.55 \\
\hline & Plant and equipment are maintained to a safe standard & 0.50 \\
\hline \multirow{6}{*}{$\mathrm{PCSK} / \mathrm{B}$} & My colleagues are familiar with the usage of safety equipments (e.g., fire extinguisher, fire hydrant) & 0.76 \\
\hline & My colleagues concern safety issues in the workplace & 0.75 \\
\hline & My colleagues are qualified with work knowledge and skills & 0.73 \\
\hline & My colleagues comply with safety procedures at all times. & 0.69 \\
\hline & My colleagues are with good safety habits (e.g., wear helmet and safety belts) & 0.67 \\
\hline & My colleagues thoroughly communicate work-related information with each other & 0.63 \\
\hline
\end{tabular}

Note: PCSK/B: perceived colleagues' safety knowledge/behavior.

Table 2

Goodness-of-fit for confirmatory factor analyses of safety climate and behavior measures.

\begin{tabular}{|c|c|c|c|c|c|c|c|}
\hline Concept & Model & $\chi^{2}$ & df & CFI & RFI & IFI & RMSEA \\
\hline PCSK/B & Single factor model & 22.93 & 9 & 0.96 & 0.90 & 0.96 & 0.06 \\
\hline Safety Climate & Single factor model & 200.31 & 74 & 0.95 & 0.90 & 0.95 & 0.07 \\
\hline \multirow[t]{2}{*}{ Safety behavior } & Single factor model & 92.96 & 9 & 0.94 & 0.85 & 0.94 & 0.12 \\
\hline & 2-factor model & 28.79 & 8 & 0.99 & 0.95 & 0.99 & 0.06 \\
\hline Measurement model & & 872.31 & 281 & 0.92 & 0.90 & 0.92 & 0.06 \\
\hline
\end{tabular}

Note: PCSK/B: perceived colleagues' safety knowledge/behavior.

for $51 \%$ variance) was larger than 1 (Table 1 ), and indices of onefactor model CFA met the requirements (Table 2). Based on that, we treated safety climate as a global factor in the following analysis which is consistent with Griffin and Neal's (2000) and Zohar and Luria's (2005) research.

\subsection{2. $P C S K / B$}

A 6-item scale was used to assess PCSK/B. An example of the items read "My colleagues comply with safety procedures at all times." Table 1 shows the EFA results with item factor loadings, and one-factor model CFA results were illustrated in Table 2. This construct was treated by single factor solution with all parameters meeting the requirements.

\subsubsection{Safety behavior}

A 6-item scale from Neal and Griffin (2006) was adopted to assess two dimensions of safety performance. Safety compliance was assessed by three items. An example item was "I use all the necessary safety equipment to do my job." Safety participation was assessed by three items too. An example item was "I voluntarily carry out tasks or activities that help to improve workplace safety." With CFA, a two-factor model of safety behavior, including safety compliance and safety participation fit the data well with all parameters meeting the requirements (Table 2), which is in consistence with Griffin and Neal's (2000) model.

The reliability of every scale was equal to or above 0.75 (Cronbach's alphas are in Table 3), which is at an accepted level. Reliability of safety compliance was 0.75 .

\subsubsection{Self-report injuries}

Employees reported whether each major body part had been injured during the past year, including head, neck, eyes, shoul- der, arms, wrist, hand, upper back, lower back, legs, ankles, feet, and other (Goldenhar et al., 2003). And then injury variable in the following analyses was a summation of the responses to these questions.

\subsubsection{Self-report near misses}

Employees were also asked to recall the total number of near misses (i.e., an incident that could have resulted in an injury but did not) that they had experienced during the past year (Goldenhar et al., 2003).

Injuries and near misses represent low base-rate and count variables, therefore, Poisson regression was often used to analyze this type of data (Hofmann and Mark, 2006). In the following Hierarchical linear modeling (HLM), Poisson distribution of outcome type was chosen to analyze the count variables.

Table 3

Descriptive, individual-level inter-correlations, and internal consistency reliability.

\begin{tabular}{lllllllll}
\hline Variable & M & SD & 1 & 2 & 3 & 4 & 5 & 6 \\
\hline 1. PCSK/B & 5.68 & 0.78 & 0.77 & & & & & \\
2. Safety climate & 4.76 & 1.00 & $0.55^{* *}$ & 0.93 & & & & \\
3. Safety compliance & 4.83 & 0.82 & $0.23^{* *}$ & 0.03 & 0.75 & & & \\
4. Safety participation & 4.33 & 0.94 & $0.25^{* *}$ & $0.13^{* *}$ & $0.63^{* *}$ & 0.78 & & \\
5. Injury & 1.97 & 2.39 & -0.06 & $-0.19^{* *}$ & 0.01 & $-0.11^{*}$ & - & \\
6. Near miss & 0.50 & 0.90 & -0.06 & $-0.16^{* *}$ & $0.13^{* *}$ & 0.08 & $0.28^{* *}$ & - \\
\hline
\end{tabular}

Note: Cronbach's alphas are in italics on the diagonal. PCSK/B: perceived colleagues' safety knowledge/behavior.

${ }^{*} p<0.05$.

$p<0.01$. 
Table 4

HLM results: effects of unit-level safety climate and PCSK/B on safety behavior.

\begin{tabular}{|c|c|c|c|c|c|c|c|c|c|c|c|}
\hline \multirow[t]{2}{*}{ Model } & \multicolumn{11}{|c|}{ Parameter estimates } \\
\hline & $\gamma_{00}$ & $\gamma_{01}$ & $\gamma_{02}$ & $\gamma_{03}$ & $\gamma_{04}$ & $\gamma_{10}$ & $\gamma_{11}$ & $\sigma^{2}$ & $\tau_{00}$ & $\tau_{11}$ & Deviance \\
\hline $\begin{array}{l}\text { Model C1 } \\
\text { L1 }: \mathrm{SBC}_{i j}=\beta_{0 j}+\beta_{1 j}\left(\mathrm{PCSK} / B_{i j}\right)+\gamma_{i j} \\
\mathrm{~L} 2: \beta_{0 j}=\gamma_{00}+\gamma_{01}(\text { UnitSC })+\gamma_{02}\left(\text { dummy }_{j}\right)+\mu_{0} \\
\text { L2 }: \beta_{1 j}=\gamma_{10}+\mu_{1} \\
\text { Model C2 }\end{array}$ & $5.26^{* * *}$ & -0.11 & $-0.92^{* * *}$ & & & $0.31^{* * *}$ & & 0.36 & $0.03^{* * *}$ & 0.01 & 1073.89 \\
\hline $\begin{array}{l}\mathrm{L} 1: \mathrm{SBC}_{i j}=\beta_{0 j}+\beta_{1 j}\left(\mathrm{PCSK} / B_{i j}\right)+\gamma_{i j} \\
\mathrm{~L} 2: \beta_{0 j}=\gamma_{00}+\gamma_{01}(\text { UnitSC }) \\
+\gamma_{02}\left(\text { dummy }_{j}\right)+\gamma_{03}(\text { Mpcskb }) \\
+\gamma_{04}(\mathrm{Mpcskb} \times \text { UnitSC })+\mu_{0} \\
\mathrm{~L} 2: \beta_{1 j}=\gamma_{10}+\gamma_{11}(\text { UnitSC })+\mu_{1} \\
\text { Model P1 }\end{array}$ & $5.25^{* * *}$ & -1.17 & $-0.91^{* * *}$ & -0.68 & 0.19 & $0.32^{* * *}$ & $0.14^{*}$ & 0.36 & $0.04^{* * *}$ & 0.00 & 1076.49 \\
\hline $\begin{array}{l}\text { L1 }: \mathrm{SBP}_{i j}=\beta_{0 j}+\beta_{1 j}\left(\mathrm{PCSK} / B_{i j}\right)+\gamma_{i j} \\
\mathrm{~L} 2: \beta_{0 j}=\gamma_{00}+\gamma_{01}(\text { UnitSC })+\gamma_{02}\left(\text { dummy }_{j}\right)+\mu_{0} \\
\text { L2 }: \beta_{1 j}=\gamma_{10}+\mu_{1} \\
\text { Model P2 }\end{array}$ & $4.62^{* * *}$ & -0.02 & $-0.68^{* * *}$ & & & $0.35^{* * *}$ & & 0.66 & $0.04^{* *}$ & 0.04 & 1386.84 \\
\hline $\begin{array}{l}\mathrm{L} 1: \mathrm{SBP}_{i j}=\beta_{0 j}+\beta_{1 j}\left(\mathrm{PCSK} / B_{i j}\right)+\gamma_{i j} \\
\mathrm{~L} 2: \beta_{0 j}=\gamma_{00}+\gamma_{01}(\text { UnitSC }) \\
+\gamma_{02}\left(\text { dummy }_{j}\right)+\gamma_{03}(\text { Mpcskb }) \\
+\gamma_{04}(\mathrm{Mpcskb} \times \text { UnitSC })+\mu_{0} \\
\mathrm{~L} 2: \beta_{1 j}=\gamma_{10}+\gamma_{11}(\text { UnitSC })+\mu_{1}\end{array}$ & $4.62^{* * *}$ & -2.15 & $-0.64^{* * *}$ & -1.89 & 0.41 & $0.38^{* * *}$ & 0.18 & 0.66 & $0.05^{* * *}$ & 0.01 & 1388.79 \\
\hline $\begin{array}{l}\text { Vote: (1) L1: Level 1; L2: Level 2. (2) PCSK/B: perc } \\
\text { participation. Mpcskb: group mean of PCSK/B; Dum } \\
\text { he better the model fits. } \\
{ }^{*} p<0.05 . \\
{ }^{* *} p<0.01 . \\
p<0.001 .\end{array}$ & $\begin{array}{l}\text { colle } \\
\text { de re }\end{array}$ & $\begin{array}{l}\text { es' saf } \\
\text { sentin }\end{array}$ & anizati & $\begin{array}{l}\text { behav } \\
\text { mem }\end{array}$ & nits & $\begin{array}{l}\text { It-leve } \\
\text { jiance }\end{array}$ & nea & $\mathrm{f}$ & $\begin{array}{l}\text { afety } \\
\text { it. The }\end{array}$ & $\begin{array}{l}\text { pliar } \\
\text { aller }\end{array}$ & $\begin{array}{l}\text { BP: safety } \\
\text { leviance is, }\end{array}$ \\
\hline
\end{tabular}

\subsection{Level of analysis}

Our theoretical model (see Fig. 1) consists of constructs both at the individual-level and unit-level, therefore HLM analyses were conducted to test the hypotheses in the current study. HLM can simultaneously test the effects of factors at different levels on individual-level outcomes (Bryk and Raudenbush, 1992). When testing incremental effects of level 2 variables, the grand-mean centering approach can lessen multi-collinearity in level 2 estimation and separate out the level 1 effects; while for cross-level interaction effects, the group-mean centering is more appropriate (Hofmann and Gavin, 1998; Hofmann et al., 2000). The concept of perceived colleagues' safety knowledge/behavior is at the individual-level, unit-level safety climate is aggregated by data collected from individuals.

\section{Results}

\subsection{Aggregation issue}

Researchers have proposed guidelines to determine whether aggregation is viable (Bliese, 2000; Zohar and Luria, 2005). Between-group variance and within-group homogeneity should meet the criteria. $R_{\mathrm{wg}(j)}$ was developed to assess within-group homogeneity. If $R_{\mathrm{wg}(j)}$ is greater than or equal to 0.70 , there is sufficient within-group agreement (James et al., 1984). $R_{\mathrm{wg}}$ of two units was lower than 0.70 , thus data from the two units were not taken into later multilevel analysis. Average $R_{\mathrm{wg}(j)}$ of the leaving units was 0.92 (from 0.70 to 0.99 ). To further assess homogeneity, intraclass correlation coefficient (ICC) was estimated. ICC2 is an estimate of the reliability of means (Bryk and Raudenbush, 1992), if ICC2 is greater than or equal to 0.70 , we can assume means are reliable indicator of work-unit scores. One way analysis of variance was conducted with unaggregated data, ICC2 $=0.92$, which indicated that safety climate exhibited significant between-group variance: $F(20,524)=12.69, p<0.001$. On the basis of above discussion, we aggregated individual-level safety perception to the unit-level safety climate.

\subsection{Bivariate relationship}

Initial support for our hypotheses can be gained by examining bivariate relationships. Hypothesis $1 \mathrm{~b}$ was supported.

PCSK/B is positively related with safety compliance $(r=0.23$, $p<0.01)$ and safety participation $(r=0.25, p<0.01)$. The relationship between unit-level safety climate and safety compliance is not as expected, which needs further analyses. Unit-level safety climate is positively related with safety participation $(r=0.13, p<0.01)$.

\subsection{HLM results}

Table 4 presents the HLM results testing the effect of unit-level safety climate and PCSK/B on safety behavior.

Hypothesis 1 predicts that PCSK/B and unit-level safety climate are positively related to safety compliance and participation. The results in Model C1 revealed that PCSK/B significantly predicted safety compliance $(\gamma=0.31, p<0.001)$, but unit-level safety climate did not show this trend $(\gamma=-0.11, p>0.05)$. The results in Model P1 revealed in PCSK/B significantly predicted safety participation $(\gamma=0.35, p<0.001)$, but unit-level safety climate showed an opposite trend $(\gamma=-0.02, p>0.05)$. This result of relationship between unit-level safety climate and safety participation seemed unreasonable, we need wait to see their real relationship after cross-level effect being examined. Therefore, Hypothesis $1 \mathrm{~b}$ was supported, but Hypothesis 1a was not supported.

Hypothesis 2 proposes unit-level safety climate moderates the effect of PCSK/B on safety behavior. The results in Model C2 revealed cross-level interaction was significant $(\gamma=0.14, p<0.05)$, which indicated unit-level safety climate moderating the relationship between PCSK/B and safety compliance. The results in Model P2 revealed cross-level interaction was marginally significant $(\gamma=0.18, p=0.07)$. Specifically, when unit-level safety climate was high, the effects of PCSK/B on safety behavior were stronger. When unit-level safety climate was low, the effects of PCSK/B on safety behavior were weaker (Figs. 2 and 3). Therefore, Hypothesis 2 was supported. 
Table 5

HLM results: effects of unit-level safety climate and PCSK/B on safety-related outcomes.

\begin{tabular}{|c|c|c|c|c|c|c|c|}
\hline \multirow[t]{2}{*}{ Model } & \multicolumn{7}{|c|}{ Parameter estimates } \\
\hline & $\gamma_{00}$ & $\gamma_{01}$ & $\gamma_{02}$ & $\gamma_{10}$ & $\gamma_{20}$ & $\gamma_{30}$ & $\tau_{00}$ \\
\hline $\begin{array}{l}\text { Model I1 } \\
\text { L1 }: E(\text { Injury } \mid \beta)=\lambda \\
\text { L1 }: \log [\lambda]=\eta\end{array}$ & & & & & & & \\
\hline $\begin{array}{l}\text { L1 }: \eta=\beta_{0}+\beta_{1}(\text { PCSK } / B) \\
\text { L2 }: \beta_{0}=\gamma_{00}+\gamma_{01}(\text { UnitSC })+\gamma_{02}\left(\text { dummy }_{j}\right)+\mu_{0} \\
\text { L2 }: \beta_{1}=\gamma_{10} \\
\text { Model I2 }\end{array}$ & $0.77^{* *}$ & 0.09 & $-0.83^{* *}$ & $-0.15^{* * *}$ & & & $0.26^{* * *}$ \\
\hline $\begin{array}{l}\mathrm{L} 1: E(\operatorname{Injury} \mid \beta)=\lambda \\
\mathrm{L} 1: \log [\lambda]=\eta \\
\mathrm{L} 1: \eta=\beta_{0}+\beta_{1}(\mathrm{PCSK} / B)+\beta_{2}(\mathrm{SBC})+\beta_{3}(\mathrm{SBP})\end{array}$ & & & & & & & \\
\hline $\begin{array}{l}\text { L2: } \beta_{0}=\gamma_{00}+\gamma_{01}(\text { UnitSC })+\gamma_{02}\left(\text { dummy }_{j}\right)+\mu_{0} \\
\text { L2: } \beta_{1}=\gamma_{10} \\
\text { L2: } \beta_{2}=\gamma_{20} \\
\text { L2: } \beta_{3}=\gamma_{30}\end{array}$ & $0.85^{* * *}$ & 0.11 & $-1.05^{* *}$ & -0.03 & $-0.14^{*}$ & $-0.18^{* * *}$ & $0.26^{* * *}$ \\
\hline $\begin{array}{l}\text { Model N1 } \\
\text { L1 }: E(\text { Near miss } \mid \beta)=\lambda \\
\text { L1 } \log [\lambda]=\eta\end{array}$ & & & & & & & \\
\hline $\begin{array}{l}\mathrm{L} 1: \eta=\beta_{0}+\beta_{1}(\mathrm{PCSK} / B) \\
\mathrm{L} 2: \beta_{0}=\gamma_{00}+\gamma_{01}(\text { UnitSC })+\gamma_{02}\left(\text { dummy }_{j}\right)+\mu_{0} \\
\mathrm{~L} 2: \beta_{1}=\gamma_{10}\end{array}$ & $-0.46^{*}$ & -0.15 & $-1.36^{* *}$ & -0.15 & & & $0.27^{* * *}$ \\
\hline $\begin{array}{l}\text { Model N2 } \\
\text { L1 }: E(\text { Nearmiss } \mid \beta)=\lambda \\
\text { L1 }: \log [\lambda]=\eta \\
\text { L1 }: \eta=\beta_{0}+\beta_{1}(\mathrm{PCSK} / B)+\beta_{2}(\mathrm{SBC})+\beta_{3}(\mathrm{SBP})\end{array}$ & & & & & & & \\
\hline $\begin{array}{l}\text { L2: } \beta_{0}=\gamma_{00}+\gamma_{01}(\text { UnitSC })+\gamma_{02}\left(\text { dummy }_{j}\right)+\mu_{0} \\
\text { L2: } \beta_{1}=\gamma_{10} \\
\text { L2: } \beta_{2}=\gamma_{20} \\
\text { L2: } \beta_{3}=\gamma_{30}\end{array}$ & -0.44 & -0.16 & $-1.37^{* *}$ & -0.13 & -0.06 & 0.07 & $0.26^{* * *}$ \\
\hline
\end{tabular}

Note: (1) L1: Level 1; L2: Level 2. (2) PCSK/B: perceived colleagues' safety knowledge/behavior; UnitSC: unit-level safety climate; SBC: safety compliance; SBP: safety participation; dummy code representing organizational membership.

${ }^{*} p<0.05$.

** $p<0.01$.

*** $p<0.001$.

The main effect of PCSK/B on safety compliance and safety participation was constantly significant (Model C2, $\gamma=0.32, p<0.001$, Model P2, $\gamma=0.38, p<0.001)$. While the main effect of unit-level safety climate on safety compliance and safety participation was not significant (Model C2, $\gamma=-1.17, p>0.05$, Model P2, $\gamma=-2.15$, $p>0.05$ ).

Hypothesis 3 proposes that safety behavior mediates the relationship between PCSK/B and safety-related outcomes. Possion distribution of outcome type was chosen in the HLM setting when analyzing injuries and near misses. We followed three-step test procedure for mediation and controlled for unit-level safety climate in the analyses (Baron and Kenny, 1986). In the first step, PCSK/B needs to be related to injuries, the result of Model I1 was

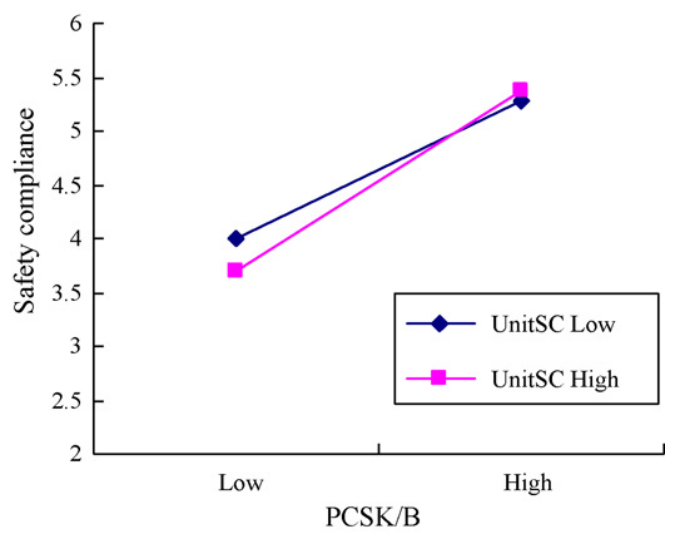

Fig. 2. Safety climate as a moderator of the relationship between PCSK/B and safety compliance. supported $(\gamma=-0.15, p<0.001)$. In the second step, PCSK/B needs to be related to safety behavior, which was supported in our testing of Hypothesis 1 above. In the third step, we included both PCSK/B and safety behavior in the regression (Model I2). We found that both safety compliance $(\gamma=-0.14, p<0.05)$ and safety participation $(\gamma=-0.18, p<0.001)$ were significantly related to injuries, and the effect of PCSK/B was not significantly related to injuries and the value was down from 0.15 to $0.03(\gamma=-0.03, p>0.05)$. So the indirect effect of safety behavior on relationship between PCSK/B and injuries exists. While for near misses outcome, Model N1, Model C1, Model P1 and Model N2 indicated that safety behavior did

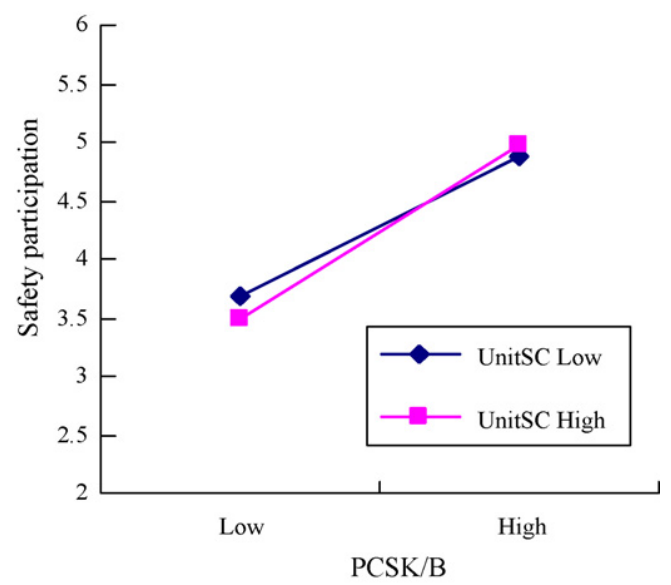

Fig. 3. Safety climate as a moderator of the relationship between PCSK/B and safety participation. 
not mediate the relationship between PCSK/B and near misses. So Hypotheses $3 \mathrm{a}$ and $3 \mathrm{~b}$ were partially supported (Table 5).

\section{Discussion}

This study tested a multilevel model based on an integrated model of safety climate-behavior-outcome relationship, covering unit-level and individual-level analysis. The results revealed that PCSK/B can predict safety behavior in the individual level. And cross-level interaction effect between unit-level safety climate and PCSK/B can predict safety behavior (safety compliance and safety participation). Furthermore, both safety compliance and participation were related to injuries. Safety behavior mediated the influence of PCSK/B on the safety outcomes.

\subsection{Theoretical implications}

Our findings regarding cross-level interaction effect on safety behavior contribute to the safety literature in several ways. First, we followed Zohar's (2002) suggestion paying attention to largely overlooked cross-level processes (for exception, see Hofmann et al., 2003) and examined the cross-level interaction of unit-level safety climate and individual PCSK/B on safety behavior Specifically, at least in Chinese context, the previously demonstrated influence of unit-level safety climate on safety behavior (Neal and Griffin, 2006; Wallace et al., 2006) is interacted with the individual-level construct PCSK/B. The unit-level safety climate cannot effectively predict individuals' safety behavior independently. We believe that the results have implications for safety research. The effect of colleagues or peer on safety issues has captured the attention of the researchers (e.g., Zohar and Tenne-Gazit, 2008), while the empirical studies related to this topic are limited so far. Our findings add to this emerging literature, suggesting that unit-level climate, and perception of colleagues' safety knowledge and behavior can have a significant impact on safety performance.

Second, our finding pertaining to the constant significant main effect of the new individual concept PCSK/B on safety behavior, indicating that employee's safety behavior are influenced by perceptions about others' beliefs and observation of others' behavior, which are consistent with the finding of social psychology: people from Eastern Asian like Chinese are more concerned with contextual factors rather than rules, thinking holistically instead of analytically (Nisbett et al., 2001; Peng et al., 2005). So just like the results obtained in other cross-cultural studies (e.g., Venkatesh and Davis, 2000), Chinese employees will concern the contextual factors such as important others (like co-workers) too. Work contextual factors have also been mentioned in Western studies, which said "Perceived safety at work was directly influenced by various work situational factors independent of safety climate" (DeJoy et al., 2004). Thus concerning the important effect of individual PCSK/B in Chinese working settings, PCSK/B might also have their potential influence on safety behavior in Western settings. In consumer study, the relationship between normative factors and behavior intentions were showing the strongest with Chinese participants (Bagozzi et al., 2000), further cross-culture study in safety field would be helpful to understand the effect of cultural factors on the norms-behavior relationship. Furthermore, Neal and Griffin (2000, Neal and Griffin, 2006) modeled that motivation and knowledge can directly influence safety behavior, so it is essential to do further research to examine the mechanism of safety motivation and knowledge on the relationship between perception of other employees' behavior influences and their own safety behavior in future.

Finally, safety behavior mediated the relationship between PCSK/B and injuries. This finding suggests that organizations should take more actions to encourage employee safety behavior, through which employees' personal health and safety can be improved. While the lacking evidence of the predictors and near misses relationship suggests that to improve workplace safety, individuallevel safety behavior is only a precursor, the most proximal variable, and there are many other underlying process variables or conditions that affect the safety outcomes. The combination of unit-level safety climate and all the colleagues' safety behavior can make a great effect on the workplace safety.

\subsection{Managerial implications}

The results of our study have several managerial implications. First, given the significant effect of PCSK/B on safety performance in current investigation, managers should consider advocating the restructure of descriptive norms to improve PCSK/B, as employees in the same unit can become one another's behavioral model. Descriptive norms approach as a kind of informal social control has been recognized valid in improving pro-environmental (Cialdini, 2007; Lapinski et al., 2007) and health behavior (Real and Rimal, 2007). Specifically, for example, offering employees opportunities to observe colleagues' safety behavior in routine tasks, establishing regular peer communication about their safety practices or any other approach which can modify perceived prevalence of safety behavior might be helpful to the improvement of safety behavior. Additionally, according to the "Hawthorne study", when employees realize that others pay attention to their behavior in daily working life, and they are important than they thought (their behavior will affect others' behavior), it is possible for them to control and improve their safety behavior, which in turn, can improve self and colleagues' safety performance.

Second, unit-level safety climate exists within the same organization, which is consistent with existent literature (Glendon and Litherland, 2001; Zohar, 2000). The effect of cross-level interaction on safety performance suggests within positive safety climate individuals are more easily influenced by PCSK/B. Therefore, organizations should pay attention to both unit-level and peer-level influences. Based on normative restructuring approach to improve $\mathrm{PCSK} / \mathrm{B}$, measurement of safety climate is thought to be a useful diagnostic tools to provide an early warning of potential safety system failure(s) (Cooper and Phillips, 2004), a proactive action Safety intervention (Siu et al., 2004). So both levels' formal safety training can provide employees opportunities to share their ideas and opinions, thus easing the way to improved climate.

In the two typical state-owned companies attending to this study, the concepts of safety climate and safety culture have just been imported in recent years. It is essential to increase the relative training to make the employees systematically understand the factors affecting safety performance, which are important preconditions to ensure the good safety performance of the whole corporation. Our findings based on state-owned companies might also benefit private companies and joint-venture companies, too. As qualitative studies suggested employees in private companies are more dependent on personal connections than employees in state owned or collective hybrid companies (Xin and Pearce, 1996). Therefore, employees in other types of companies may even more influenced by PCSK/B. Future research should examine whether our findings can be replicated in private or international companies.

\subsection{Limitations}

The study had several limitations. One of the limitations was the cross-sectional design, which prevents drawing any causal inferences. It is possible that employees experienced injuries might show high safety performance (Clarke, 2006). Only the longitudinal design can help to explain it. 
A second limitation concerns that dependent variables were self-reported behaviors, injuries and number of near misses. The results might be criticized for common method bias. For safety researches, Hofmann and Stetzer (1996) stated that variance on safety-related outcomes would be suppressed. People would probably tend to underreport injuries and near misses due to social desirability. Thus the relationship between these variables and their predictors would be attenuated (Probst and Brubaker, 2001). And in order to examine whether the bias would account for all the relationships among the variables, we followed Harman's single factor test (Podsakoff et al., 2003), which is checking if all the items involved belonging to one factor, and if it is the case, the significant relationship could be attributed to the bias, or it can be concluded that the significant relationship are real to some extent. The unrotated exploratory factor analysis showed one factor did not account for the majority of the covariance. Thus, common method bias could not account for all the relationships among variables. Future studies can try to get multi-resources data, such as objective records, behavioral observation to examine and validate the suggested relationships of present study.

A third limitation concerns the unit-level sample. We sampled 23 units, which were relatively small in HLM studies. The limited unit sample could potentially impact the generalizability of our findings. Kreft (as cited in Hofmann, 1997) suggested a 30/30 rule of thumb in HLM studies, however, this sample size is greater than what is typically seen in organizational research (Scandura and Williams, 2000), and in many organizational studies there are typically less than 30 higher level units in a study (Scherbaum and Ferreter, 2009). Moreover, there seems to be a tradeoff between sample sizes of Level 2 and Level 1. Hofmann (1997) stated: "If a large number of groups is present, then the number of observations required per group is reduced. Conversely, with fewer groups, one needs more individuals within each group to obtain sufficient power." With the limited units (23), we tried to get more observations, and finally obtained 631 valid data, with an average 27.4 observations per group. Due to the sample limitation at unit level, the results reported here were only based on generalized least squares (GLS) standard errors. As researchers suggested higher levels of power are achieved with larger samples at Level 2 than at Level 1, it is very essential to collect more data over more groups (Scherbaum and Ferreter, 2009).

\section{Conclusion}

The study presents a multilevel model based on an integrated model of safety climate-behavior-outcome relationship. The findings of the current study suggest that the effect of PCSK/B on safety behavior is moderated by unit-level safety climate. PCSK/B can more effectively predict safety behavior in positive safety climate than negative safety climate. The influence of PCSK/B on injuries is operating through safety behavior. Safety-priority organizations should not only solid their safety climate but also focus on the social interaction between employees to facilitate their safety behavior.

\section{Acknowledgments}

This work was supported in part by the National Natural Science Foundation of China under Grant 70401018 and 70501035, and in part by the Knowledge Innovation Project of the Chinese Academy of Sciences under Grant KSCX2-YW-R-130.

\section{References}

Ashforth, B.E., 1985. Climate formation: issues and extensions. Acad. Manage. Rev. $10(4), 837-847$.
Bagozzi, R.P., Wong, N., Abe, S., Bergami, M., 2000. Cultural and situational contingencies and the theory of reasoned action: application to fast food restaurant consumption. J. Consum. Psychol. 9 (2), 97-106.

Barling, J., Loughlin, C., Kelloway, E.K., 2002. Development and test of a model linking safety-specific transformational leadership and occupational safety. J. Appl. Psychol. 87 (3), 488-496.

Baron, R.M., Kenny, D.A., 1986. The moderator-mediator variable distinction in social psychological research: conceptual, strategic, and statistical considerations. J. Pers. Soc. Psychol. 51 (6), 1173-1182.

Bliese, P.D., 2000. Within-group agreement, non-independence, and reliability: implications for data aggregation and analysis. In: Klein, K.J., Kozlowski, S.W. (Eds.), Multilevel Theory, Research, and Methods in Organizations: Foundations, Extensions, and New Directions. Jossey-Bass, San Francisco, pp. 349-381.

Brislin, R.W., 1980. Translation and content analysis of oral and written material. Handb. Cross Cult. Psychol. 2, 389-444.

Bryk, A.S., Raudenbush, S.W., 1992. Hierarchical Linear Models: Applications and Data Analysis Methods. Sage, Newburry Park, CA.

Burnkrant, R.E., Cousineau, A., 1975. Informational and normative social influence in buyer behavior. J. Consum. Res. 2 (3), 206.

Cialdini, R.B., 2007. Descriptive social norms as underappreciated sources of social control. Psychometrika 72 (2), 263-268.

Cialdini, R.B., Raymond, R., 1990. A focus theory of normative conduct: recycling the concept of norms to reduce littering in public places. J. Pers. Soc. Psychol. 58 (6), 1015-1026.

Clarke, S., 1999. Perceptions of organizational safety: implications for the development of safety culture. J. Organ. Behav. 20 (2), 185-198.

Clarke, S., 2006. The relationship between safety climate and safety performance: a meta-analytic review. J. Occup. Health Psychol. 11 (4), 315-327.

Clarke, S., Ward, K., 2006. The role of leader influence tactics and safety climate in engaging employees' safety participation. Risk Anal. 26 (5), 1175-1185.

Cooper, M.D., Phillips, R.A., 2004. Exploratory analysis of the safety climate and safety behavior relationship. J. Safety Res. 35 (5), 497-512.

Cox, S., Flin, R., 1998. Safety culture: philosopher's stone or man of straw? Work Stress 12 (3), 189-201.

DeJoy, D.M., Schaffer, B.S., Wilson, M.G., Vandenberg, R.J., Butts, M.M., 2004. Creating safer workplaces: assessing the determinants and role of safety climate. J. Safety Res. 35 (1), 81-90.

Deutsch, M., Gerard, H.B., 1955. A study of normative and informational social influences upon individual judgment. J. Abnorm. Soc. Psychol. 51 (3), 629-636.

Ehrhart, M.G., Naumann, S.E., 2004. Organizational citizenship behavior in work groups: a group norms approach. J. Appl. Psychol. 89 (6), 960-974.

Evans, B., Glendon, A.I., Creed, P.A., 2007. Development and initial validation of an aviation safety climate scale. J. Safety Res. 38 (6), 675-682.

Fahlbruch, B., Wilpert, B., 1999. System safety-an emerging field for i/o psychology. Int. Rev. Ind. Organ. Psychol. 14, 55-93.

Flin, R., Mearns, K., O'Connor, P., Bryden, R., 2000. Measuring safety climate: identifying the common features. Safety Sci. 34 (1), 177-192.

Glendon, A.I., Litherland, D.K., 2001. Safety climate factors, group differences and safety behaviour in road construction. Safety Sci. 39 (3), 157-188.

Glendon, A.I., Evans, B., 2007. Safety climate in australian railways. In: Wilson, J., Norris, B., Clarke, T., Mills, A. (Eds.), People and Rail Systems: Human Factors at the Heart of the Railway. Ashgate, United Kingdom, pp. 409-417.

Goldenhar, L.M., Williams, L.J., Swanson, N.G., 2003. Modelling relationships between job stressors and injury and near-miss outcomes for construction labourers. Work Stress 17 (3), 218-240.

Gonzalez-Roma, V., Peiro, J.M., Tordera, N., 2002. An examination of the antecedents and moderator influences of climate strength. J. Appl. Psychol 87 (3), 465-473.

Griffin, M.A., Neal, A., 2000. Perceptions of safety at work: a framework for linking safety climate to safety performance, knowledge, and motivation. J. Occup. Health Psychol. 5 (3), 347-358.

Hetherington, C., Flin, R., Mearns, K., 2006. Safety in shipping: the human element. J. Safety Res. 37 (4), 401-411.

Hofmann, D.A., 1997. An overview of the logic and rationale of hierarchical linear models. J. Manage. 23 (6), 723-744.

Hofmann, D.A., Gavin, M.B., 1998. Centering decisions in hierarchical linear models: implications for research in organizations. J. Manage. 24 (5), 623-641.

Hofmann, D.A., Jacobs, R., Landy, F., 1995. High reliability process industries: individual, micro, and macro organizational influences on safety performance. J. Safety Res. 26 (3), 131-149.

Hofmann, D.A., Mark, B., 2006. An investigation of the relationship between safety climate and medication errors as well as other nurse and patient outcomes. Pers. Psychol. 59 (4), 847-869.

Hofmann, D.A., Morgeson, F.P., 1999. Safety-related behavior as a social exchange: the role of perceived organizational support and leader-member exchange. J. Appl. Psychol. 84 (2), 286-296.

Hofmann, D.A., Morgeson, F.P., Gerras, S.J., 2003. Climate as a moderator of the relationship between leader-member exchange and content specific citizenship: safety climate as an exemplar. J. Appl. Psychol. 88 (1), 170-178.

Hofmann, D.A., Stetzer, A., 1996. A cross-level investigation of factors influencing unsafe behaviors and accidents. Pers. Psychol. 49 (2), 307-339.

Hofmann, D.A., Griffin, M.A., Gavin, M.B., 2000. The application of hierarchical linear modeling to organizational research. In: Klein, K.J., Kozlowski, S.W.(Eds.), Multilevel Theory, Research, and Methods in Organizations: Foundations, Extensions, and New Directions. Jossey-Bass, San Francisco, CA, pp. 467-511.

James, L.R., Demaree, R.G., Wolf, G., 1984. Estimating within-group interrater reliability with and without response bias. J. Appl. Psychol. 69 (1), 85-98. 
Kallgren, C.A., Reno, R.R., Cialdini, R.B., 2000. A focus theory of normative conduct: when norms do and do not affect behavior. Pers. Soc. Psychol. Bull. 26 (8), 1002

Klein, K.A., Boster, F.J., 2006. Subjective, descriptive and injunctive norms: three separate constructs. In: Presented at the Annual Meeting of the International Communication Association, Dresden International Congress Centre, Dresden, Germany.

Kozlowski, S.W., Klein, K.J., 2000. A multilevel approach to theory and research in organizations: contextual, temporal, and emergent processes. In: Klein, K.J. Kozlowski, S.W. (Eds.), Multilevel Theory, Research, and Methods in Organizations: Foundations, Extensions, and New Directions. Jossey-Bass, San Francisco, pp. 3-90.

Lapinski, M.K., Rimal, R.N., 2005. An explication of social norms. Commun. Theory $15(2), 127-147$

Lapinski, M.K., Rimal, R.N., Devries, R., Lee, E.L., 2007. The role of group orientation and descriptive norms on water conservation attitudes and behaviors. Health Commun. 22 (2), 133-142.

Markus, H.R., Kitayama, S., 1991. Culture and the self: implications for cognition, motivation, and emotion. Psychol. Rev. 98, 224-253.

McDonald, N., Corrigan, S., Daly, C., Cromie, S., 2000. Safety management systems and safety culture in aircraft maintenance organisations. Safety Sci. 34 (1-3), 151-176.

Mearns, K.J., Flin, R., 1999. Assessing the state of organizational safety-culture or climate? Curr. Psychol. 18 (1), 5-17.

Neal, A., Griffin, M.A., 2006. A study of the lagged relationships among safety climate, safety motivation, safety behavior, and accidents at the individual and group levels. J. Appl. Psychol. 91 (4), 946-953.

Nisbett, R.E., Peng, K., Choi, I., Norenzayan, A., 2001. Culture and systems of thought: holistic versus analytic cognition. Psychol. Rev. 108 (2), 291-310.

Oyserman, D., Coon, H.M., Kemmelmeier, M., 2002. Rethinking individualism and collectivism: evaluation of theoretical assumptions and meta-analyses. Psychol. Bull. 128 (1), 3-72.

Park, H.S., Levine, T.R., 1999. The theory of reasoned action and self-construal: evidence from three cultures. Commun. Monogr. 66 (3), 199-218.

Peng, K., Spencer-Rodgers, J., Zhong, N., 2005. Naïve dialecticism and the tao of chinese thought. In: Kim, U., Yang, K., Huang, G. (Eds.), The Handbook of Indigenous and Cultural Psychology. Blackwell, pp. 247-262.

Pidgeon, N., 1998. Safety culture: key theoretical issues. Work Stress 12 (3), 202-216.

Podsakoff, P.M., Mackenzie, S.B., Lee, J.Y, Podsakoff, N.P. 2003. Common method biases in behavioral research: A critical review of the literature and recommended remedies. J. Appl. Psychol. 88 (5), 879-903.

Probst, T.M., Brubaker, T.L., 2001. The effects of job insecurity on employee safety outcomes: cross-sectional and longitudinal explorations. J. Occup. Health Psychol. 6 (2), 139-159.

Real, K., Rimal, R.N., 2007. Friends talk to friends about drinking: exploring the role of peer communication in the theory of normative social behavior. Health Commun. 22 (2), 169-180.

Reason, J., 1990. Human Error. Cambridge University Press.

Rashotte, L.S., 2007. Social influence. In: Ritzer, G. (Ed.), The Blackwell Encyclopedia of Sociology. Blackwell Publishing, pp. 4426-4429.
Rogers, E.M., 1986. Communication Technology: The New Media in Society. The Free Press New York, NY, USA.

Scandura, T.A., Williams, E.A., 2000. Research methodology in management: current practices, trends, and implications for future research. Acad. Manage. J. 43 (6), $1248-1264$.

Scherbaum, C.A., Ferreter, J.M., 2009. Estimating statistical power and required sample sizes for organizational research using multilevel modeling. Organ. Behav. Methods 12 (2), 347-367.

Seo, D.C., 2005. An explicative model of unsafe work behavior. Safety Sci. 43 (3), 187211.

Shannon, H.S., Mayr, J., Haines, T., 1997. Overview of the relationship between organizational and workplace factors and injury rates. Safety Sci. 26 (3), 201-217.

Siu, O.L., Phillips, D.R., Leung, T.W., 2004. Safety climate and safety performance among construction workers in hong kong. The role of psychological strains as mediators. Accid. Anal. Prev. 36 (3), 359-366.

Smith, C.A., Organ, D.W., Near, J.P., 1983. Organizational citizenship behavior: its nature and antecedents. J. Appl. Psychol. 68 (4), 653-663.

State Administration of Work Safety. The loss of accidents cost China's economy an estimated 2\% GDP. <http://news.sina.com.cn/c/2005-0614/17556170809s.shtml> (retrieved 14.06.05).

State Administration of Work Safety. The statistics of injuries and deaths in accidents in 2006. <http://www.chinasafety.gov.cn/newpage/ Contents/Channel_4181/2007/0111/836/content_836.htm> (retrieved 11.01.07).

Venkatesh, V., Davis, F.D., 2000. A theoretical extension of the technology acceptance model: four longitudinal field studies. Manage. Sci. 46 (2), 186-204.

Wallace, J.C., Popp, E., Mondore, S., 2006. Safety climate as a mediator between foundation climates and occupational accidents: a group-level investigation. J. Appl. Psychol. 91 (3), 681-688.

Wittenbrink, B., Henly, J.R., 1996. Creating social reality: informational social influence and the content of stereotypic beliefs. Pers. Soc. Psychol. Bull. 22 (6), 598-610.

Xin, K.R., Pearce, J.L., 1996. Guanxi: connections as substitutes for formal institutional support. Acad. Manage. J. 39 (6), 1641-1658.

Zohar, D., 1980. Safety climate in industrial organizations: theoretical and applied implications. J. Appl. Psychol. 65 (1), 96-102.

Zohar, D., 2000. A group-level model of safety climate: testing the effect of group climate on microaccidents in manufacturing jobs. J. Appl. Psychol. 85 (4) 587-596.

Zohar, D., 2002. The effects of leadership dimensions, safety climate, and assigned priorities on minor injuries in work groups. J. Organ. Behav. 23 (1), 75-92.

Zohar, D., 2008. Safety climate and beyond: a multi-level multi-climate framework. Saf. Sci. 46 (3), 376-387.

Zohar, D., Luria, G., 2005. A multilevel model of safety climate: cross-level relationships between organization and group-level climates. J. Appl. Psychol. 90 (4), 616-628.

Zohar, D., Tenne-Gazit, O., 2008. Transformational leadership and group interaction as climate antecedents: a social network analysis. J. Appl. Psychol. 93 (4), 744-757. 